\section{Meta-analysis quantifies the effect of obesity on the risk of kidney disease}

Evidence is accumulating that obesity might be an important risk factor for the development and progression of kidney disease. To comprehensively assess the association between obesity and kidney disease, Wang et al. performed a meta-analysis of 16 cohort studies conducted in the general population.

The studies were identified from a search of the PubMed database. The studies reported on 19 cohorts -12 from the US, 4 from Europe, 2 from Japan and 1 from Korea. The median follow-up duration was 15 years (range 3-35 years). Estimated pooled relative risks (RRs) revealed that, compared with normalweight individuals (BMI 18.5-24.9 kg/m²), overweight individuals (BMI $25-29.9 \mathrm{~kg} / \mathrm{m}^{2}$ ) had a considerably increased risk of kidney disease (RR 1.40, 95\% Cl 1.30-1.50). The RR of kidney disease in obese individuals (BMI $\geq 30 \mathrm{~kg} / \mathrm{m}^{2}$ ) was even greater (RR $1.83,95 \%$ $\mathrm{Cl}$ 1.57-2.13). When BMI was treated as a continuous variable, each $1 \mathrm{~kg} / \mathrm{m}^{2}$ increment in $\mathrm{BMI}$ increased the risk of kidney disease by $6 \%$ (pooled RR 1.06, 95\% Cl 1.05-1.07). Stratification of the data by sex revealed a stronger association between obesity and kidney disease in women than in men (odds ratio $1.92,95 \% \mathrm{Cl} 1.78-2.07$, vs $1.49,95 \% \mathrm{Cl}$ 1.36-1.63). Population-attributable risk calculations for the US indicated that approximately $24.2 \%$ of kidney disease cases in men and $33.9 \%$ of cases in women could be attributable to obesity and being overweight.

Original article Wang Y et al. (2008) Association between obesity and kidney disease: a systematic review and metaanalysis. Kidney Int 73: 19-33

\section{Systematic review validates the RIFLE criteria in acute kidney injury}

The classification system for acute kidney injury (AKI) proposed by the Acute Dialysis Quality Initiative workgroup, known as RIFLE (Risk, Injury, Failure, Loss, and End-stage kidney disease), is intended to facilitate stratification of the AKI population according to disease severity. Since the publication of the RIFLE system in 2004 , a number of studies have been conducted to validate this approach in clinical practice. Ricci et al. have examined the outcomes of these studies in a systematic review of literature published from August 2004 to June 2007.

In total, 24 studies were identified in which the RIFLE criteria were used to define the severity of AKI. Among these studies, 13 reported patient-level data on mortality in both patients with and those without AKI. Pooled relative risk estimates showed that the likelihood of death increased with worsening RIFLE class. In comparison with patients who did not have AKI, the relative risks of mortality in the Risk, Injury, and Failure groups were 2.40, 4.15 and 6.37 , respectively $(P<0.0001$ for all comparisons). Although there was significant heterogeneity among the studies, the general correlation between worsening RIFLE class and increasing risk of mortality held true across a diverse range of AKI populations, including individuals undergoing cardiac surgery and critically ill patients.

The authors conclude that the RIFLE classification system is a useful clinical tool that can successfully stratify patients according to AKI severity.

Original article Ricci Z et al. (2008) The RIFLE criteria and mortality in acute kidney injury: a systematic review. Kidney Int 73: 538-546

\section{Does hyperechogenicity of the renal cortex indicate kidney disease in children?}

In adults and in children older than 2-3 months, the renal cortex is normally less echogenic than the liver parenchyma. Some researchers have suggested that hyperechogenicity of the renal cortex (i.e. renal cortex echogenicity greater than or equal to liver echogenicity) indicates renal disease, but Wiersma et al. report that such a finding is transient, nonspecific and not necessarily predictive of renal disease in children.

The study included 189 children (aged 2-15 years, mean age 10 years) with acute abdominal illness who underwent sonographic examination. Patients with a history of renal disease or a recent episode of pyelonephritis were excluded. During sonography, renal cortex echogenicity was classified as less than, equal to, or greater than liver 